\title{
Cuidados de enfermagem aos pacientes portadores de câncer colorretal
}

\author{
Nursing care for colorectal cancer patients \\ Atención de enfermería para pacientes con cáncer colorrectal
}

Recebido: 14/07/2021 | Revisado: 19/07/2021 | Aceito: 22/07/2021 | Publicado: 31/07/2021

\author{
Ana Luíza Chaves Silva \\ ORCID: https://orcid.org/0000-0001-8889-8772 \\ Instituto Brasileiro de Medicina e Reabilitação, Brasil \\ E-mail: analuchaves98@ outlook.com \\ Leonardo Jorge da Silva \\ ORCID: https://orcid.org/0000-0003-3005-212X \\ Instituto Brasileiro de Medicina e Reabilitação, Brasil \\ E-mail: lehosoibelman@gmail.com \\ Amanda Sousa Oliveira \\ ORCID: https://orcid.org/0000-0003-1616-0765 \\ Instituto Brasileiro de Medicina e Reabilitação, Brasil \\ E-mail: amandaols917@gmail.com \\ Rafaela Lima de Oliveira \\ ORCID: https://orcid.org/0000-0002-0994-3246 \\ Instituto Brasileiro de Medicina e Reabilitação, Brasil \\ E-mail: rafaela.limaoliveira@yahoo.com.br \\ Luciano Godinho Almuinha Ramos \\ ORCID: https://orcid.org/0000-0001-9958-3151 \\ Instituto Brasileiro de Medicina e Reabilitação, Brasil \\ E-mail: lucianogodinho@yahoo.com.br
}

\begin{abstract}
Resumo
Este estudo disserta sobre os cuidados de enfermagem aos pacientes portadores de câncer colorretal. Tem como objetivo destacar os cuidados de enfermagem em pacientes portadores de câncer colorretal. Para isso, foi utilizada a seguinte questão norteadora: Quais são os principais cuidados de enfermagem necessários aos pacientes portadores de câncer colorretal? Como critérios de inclusão foram utilizados artigos científicos entre os anos de 2016 a 2020, nas bases de dados, LILACS, BVS, SCIELO e PUBMED, em língua Inglesa, Francesa e Espanhola. Como critérios de exclusão foram excluídos artigos fora do espaço temporal de pesquisa e que não abordavam a temática. Os resultados obtidos através dos 20 artigos científicos selecionados, trouxeram efetivação a elaboração de três categorias temáticas: Os principais cuidados de enfermagem ao paciente com câncer colorretal; Qualidade de vida dos pacientes ostomizados portadores de câncer colorretal; A importância da educação em saúde nos pacientes com câncer colorretal. Como conclusão, foi ressaltada a importância do enfermeiro como educador em saúde e da Sistematização da Assistência de Enfermagem no acompanhamento ao paciente portador de câncer colorretal desde o diagnóstico, até a alta hospitalar. Também foi destacada a importância da criação de redes de apoio para as pessoas acometidas com esta doença e de como os profissionais de saúde precisam estar capacitados e atualizados para suprir as necessidades destes pacientes em todos os âmbitos de suas vidas.
\end{abstract}

Palavras-chave: Câncer colorretal; Cuidados de enfermagem; Educação em saúde.

\begin{abstract}
This study discusses nursing care for patients with colorectal cancer. It aims to highlight nursing care for patients with colorectal cancer. For this, the following guiding question was used: What is the main nursing care necessary for patients with colorectal cancer? As inclusion criteria, scientific articles between the years 2016 to 2020 were used, in the databases, LILACS, BVS, SCIELO and PUBMED, in English, French and Spanish. As exclusion criteria, articles outside the research time frame and that did not address the theme were excluded. The results obtained through the 20 selected scientific articles brought about the elaboration of three thematic categories: The main nursing care for patients with colorectal cancer; Quality of life of ostomized patients with colorectal cancer; The importance of health education in patients with colorectal cancer. In conclusion, the importance of the nurse as a health educator and the Systematization of Nursing Care in monitoring the patient with colorectal cancer from diagnosis to hospital discharge was highlighted. It was also highlighted the importance of creating support networks for people affected with this disease and how health professionals need to be trained and updated to meet the needs of these patients in all areas of their lives.
\end{abstract}

Keywords: Colorectal cancer; Nursing care; Health education.

\section{Resumen}

Este estudio analiza la atención de enfermería para pacientes con cáncer colorrectal. Tiene como objetivo destacar la atención de enfermería a los pacientes con cáncer colorrectal. Para ello se utilizó la siguiente pregunta orientadora: 
¿Cuáles son los principales cuidados de enfermería necesarios para los pacientes con cáncer colorrectal? Como criterios de inclusión se utilizaron artículos científicos entre los años 2016 a 2020, en las bases de datos LILACS, BVS, SCIELO y PUBMED, en inglés, francés y español. Como criterio de exclusión, se excluyeron los artículos fuera del marco temporal de la investigación y que no abordaran el tema. Los resultados obtenidos a través de los 20 artículos científicos seleccionados propiciaron la elaboración de tres categorías temáticas: Los principales cuidados de enfermería al paciente con cáncer colorrectal; Calidad de vida de pacientes ostomizados con cáncer colorrectal; La importancia de la educación sanitaria en pacientes con cáncer colorrectal. En conclusión, se destacó la importancia de la enfermera como educadora en salud y la Sistematización de la Atención de Enfermería en el seguimiento del paciente con cáncer colorrectal desde el diagnóstico hasta el alta hospitalaria. También se destacó la importancia de crear redes de apoyo a las personas afectadas por esta enfermedad y cómo los profesionales de la salud deben estar capacitados y actualizados para satisfacer las necesidades de estos pacientes en todos los ámbitos de su vida.

Palabras clave: Cáncer colorrectal; Cuidado de enfermera; Educación para la salud.

\section{Introdução}

De acordo com Brasil (2021), o câncer de intestino abrange os tumores que se iniciam na parte do intestino grosso chamada cólon, reto e ânus, também conhecido como câncer colorretal. Segundo Pullig, et al. (2019), o câncer colorretal tem como fatores de risco questões relacionadas à predisposição genética, acometimento do cólon por doenças inflamatórias e ao estilo de vida, principalmente alimentação inadequada, sedentarismo, tabagismo, consumo excessivo de álcool, alta ingesta de carnes processadas e/ou vermelhas; e esses hábitos variam de acordo com as condições socioeconômicas. Pacientes com doenças no trato gastrointestinal devem ter acompanhamento individualizado, como por exemplo a doença de Crohn, que é uma doença inflamatória crônica e pode atingir qualquer segmento do trato gastrointestinal. Esta patologia tem sido associada a um risco aumentado de várias neoplasias dentre as quais, o carcinoma colorretal e constitui a principal causa de morte por cancro do aparelho digestivo. (Santos, 2017)

Segundo Bremer, et al. (2020), o desenvolvimento do câncer colorretal se inicia com a ocorrência de mutações nas células do epitélio intestinal, que ocasionam vantagens proliferativas, a proliferação aumentada origina a formação de pólipos adenomatosos benignos, que podem evoluir para a gênese de tumores malignos. O tempo de evolução entre o adenoma e o desenvolvimento de câncer é de cerca de 10 anos, assim como os demais, esse tipo de câncer pode ser prevenido com a manutenção do peso corporal adequado, a prática de atividade física, assim como a alimentação saudável, além de não fumar e não se expor ao tabagismo.

O estudo de Moura, et al. (2020), diz que os sintomas relacionados dependerão da localização do câncer e da gravidade do caso. De acordo com Brasil (2021), a detecção precoce do câncer é uma estratégia para encontrar um tumor numa fase inicial e, assim, possibilitar maior chance de tratamento. O diagnóstico requer biópsia e pode ser feito por meio da investigação com exames clínicos, laboratoriais ou radiológicos. Esse tipo de tumor pode ser detectado precocemente através de pesquisa de sangue oculto nas fezes, colonoscopia ou retossigmoidoscopia, a colonoscopia representa o padrão-ouro para detecção de lesões précancerígenas em risco de progressão para neoplasia colorretal. (Diger, et al. 2019)

Segundo Brasil (2021), o tratamento depende principalmente do tamanho, localização e extensão do tumor, nesse caso, o câncer de intestino é uma doença tratável e frequentemente curável. A cirurgia é o tratamento inicial, retirando a parte do intestino afetada e os gânglios linfáticos no abdômen. Novos protocolos e estratégias pré e perioperatórios são desenvolvidas continuamente, visando a mais rápida recuperação pós-operatória e a alta hospitalar precoce, além de diminuir os índices de morbimortalidade e de complicações cirúrgicas. Outras etapas do tratamento incluem a radioterapia, associada ou não à quimioterapia, para diminuir a possibilidade de retorno do tumor. Quando a doença está espalhada, com metástases para o fígado, pulmão ou outros órgãos, as chances de cura ficam reduzidas. (Reis, et al. 2019)

Vários teóricos que investigam o cuidado de Enfermagem, em distintos contextos, sob diferentes perspectivas e pressupostos filosóficos, são uníssonos em concordar que, apesar do cuidar ser inerente a todo ser humano, há uma especificidade no cuidado de Enfermagem, que o diferencia daquele que pode ser realizado por qualquer pessoa. Quando se fala em 
Enfermagem, reporta-se para as necessidades do ser humano, visto que essa área se propõe a trabalhar para ajudar os sujeitos a manterem ou reestabelecerem essas necessidades básicas da vida. Entretanto, como essas são muitas, é pertinente que a Enfermagem compreenda o que lhe cabe nesse amplo processo e como pode contribuir, naquilo que é preciso conhecer teórica e empiricamente o que caracteriza esse cuidado de Enfermagem, por meio do qual se pode intervir no estado de saúde/doença do sujeito, em nível individual e coletivo. (Barbosa, 2021)

Esse conceito remete ao estudo de Remor, et al. (1986), sobre a Teoria do Autocuidado de Dorothea Orem, o qual acredita que o profissional de enfermagem juntamente com o paciente, deve identificar déficits de capacidade no atendimento das necessidades individuais de autocuidado, procurando desenvolver nestes indivíduos os potenciais já existentes para tal prática. Sob um esquema simplificado, pode-se dizer que os enfermeiros "ensinam" e os pacientes "aprendem" os princípios básicos do autocuidado e suas respectivas aplicações. Entretanto, tratando-se de pacientes adultos, a incorporação de novos saberes se dá de forma um pouco mais complexa, pois os adultos aprendem predominantemente a partir do repertório de conhecimentos que já acumularam, o que significa dizer que a história de vida, os hábitos pessoais e coletivos, o imaginário popular, as crenças e os valores culturais dos pacientes atuam como elementos de referência crítica para a seleção, aceitação, modificação e aplicação de novos saberes. (Mesquita, et al. 2016)

Desta forma, o profissional de Enfermagem atua no autocuidado como regulador do sistema. Ele identifica os déficits de competência em relação à demanda de autocuidado, faz pelo indivíduo aquilo que ele não pode fazer, ensina, orienta e promove o desenvolvimento das capacidades do indivíduo para que ele possa se tornar independente da assistência de enfermagem, assumindo seu autocuidado. Estas capacidades podem se desenvolver no dia a dia, através de um espontâneo processo de aprendizagem, auxiliado pela curiosidade intelectual, pela instrução e supervisão de outros ou pela experiência na execução de medidas de autocuidado. A condição que justifica a existência da enfermagem para o indivíduo adulto é a ausência da capacidade de manter continuamente aquela quantidade e qualidade de autocuidado que é terapêutica na manutenção da vida e da saúde, na recuperação, após a doença ou dano, ou a maneira de enfrentar seus efeitos. (Remor, et al. 1986)

Diante disso, este trabalho tem o intuito de destacar os principais cuidados de enfermagem voltados para a assistência a essa patologia, que são de extrema importância para prevenção e acompanhamento da clientela submetida a esse agravo.

\section{Metodologia}

Segundo Pereira, et al. (2018), uma das principais características do conhecimento científico é a sua estruturação, pois consiste num saber ordenado, o qual é construído a partir de um conjunto de ideias. Outra característica do conhecimento científico é ser verificável, isto é, determinada ideia deve ser verificada e comprovada sob a perspectiva da ciência para que possa fazer parte do conhecimento científico.

Para alcançar o objetivo proposto, utilizou-se uma revisão integrativa da literatura. Para o levantamento dos artigos foi realizada uma busca online nos bancos de dados BVS, LILACS, SCIELO e PUBMED. Todo o processo de desenvolvimento da pesquisa e seleção dos artigos foi conduzido por quatro pesquisadores independentes, elegendo-se assim, a seguinte questão norteadora: quais são os cuidados de enfermagem relacionados ao paciente portador de câncer colorretal?

Como critérios de inclusão, foram utilizados artigos científicos entre os anos de 2016 a 2020, artigos disponíveis na íntegra e originais que abrangessem os objetivos propostos e abordassem o tema: câncer colorretal, cuidados de enfermagem e educação em saúde, totalizando 91 artigos.

Conforme os critérios de exclusão, os artigos que não apresentavam a temática, artigos de revisão, artigos que não se relacionavam aos objetivos desta pesquisa, dissertações ou teses, foram descartados. Dessa forma foram excluídos um total de 71 artigos. Sendo que, após a leitura, selecionou-se apenas 20 artigos para a elaboração final do estudo pelos bancos de dados. A figura a seguir, apresenta como foi realizada a seleção dos artigos que compuseram a amostra final da revisão. 
Figura 1. Fluxograma de busca e seleção dos 18 artigos. Rio de janeiro, RJ, Brasil, 2021.

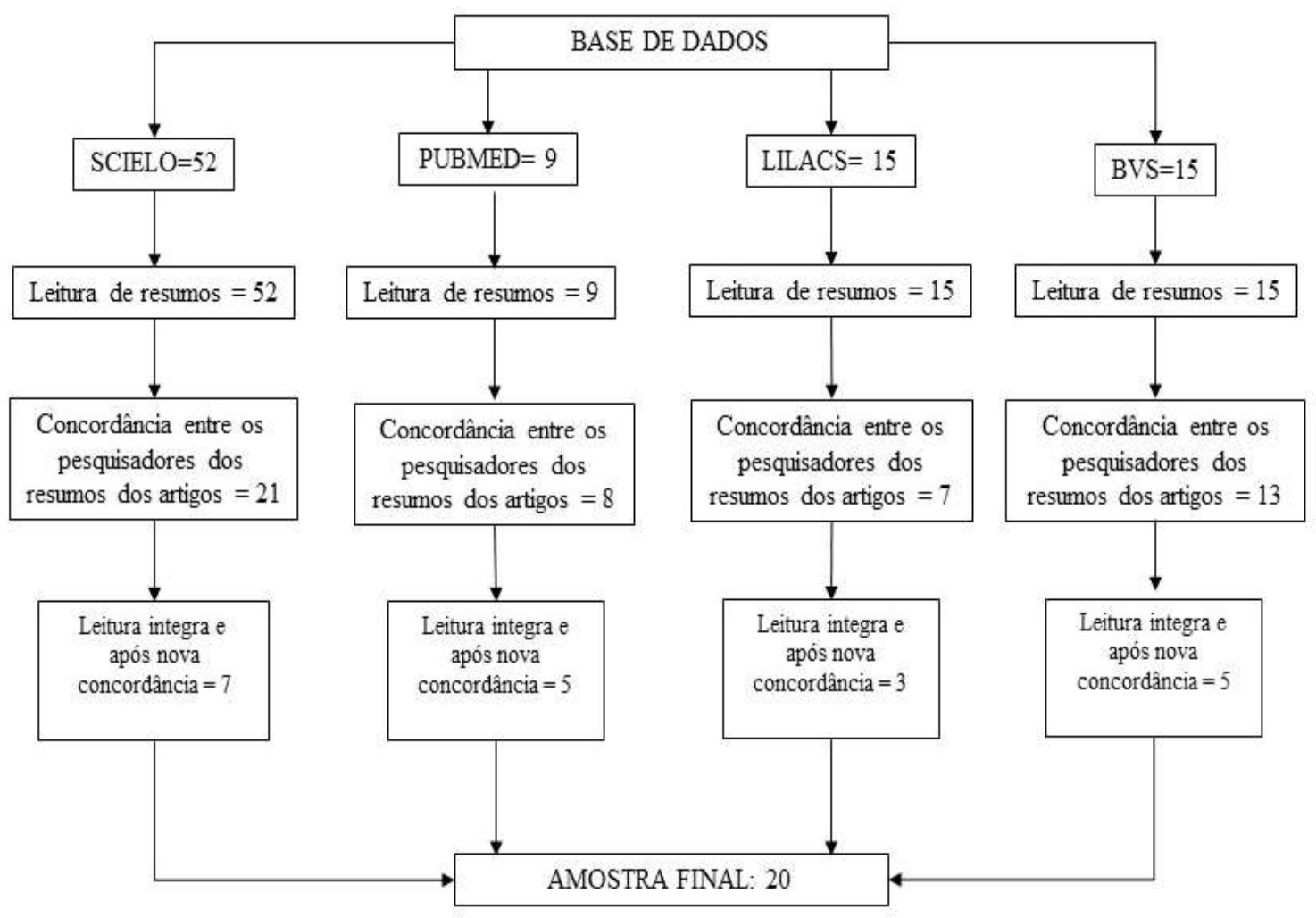

Fonte: Dados da pesquisa.

Analisando o Fluxograma, podemos observar os artigos que levaram a elaboração desta revisão, juntamente com a ordem de busca dos mesmos, verificando, banco de dados, seleção, exclusão e escolha para a obtenção da amostra final.

\section{Resultados e Discussão}

A busca dos artigos científicos foi realizada entre abril e maio de 2021, e para a apresentação das etapas de seleção dos artigos de acordo com as informações adquiridas, foi elaborado um quadro como metodologia com os principais dados dos artigos científicos escolhidos.

Dos 20 artigos selecionados no campo deste estudo, todos tiveram publicação entre 2016 e 2020, sendo em maior quantidade as apresentações dos artigos de 2019 e 2020.

Analisando o Quadro 1, podemos observar o esqueleto de organização e estrutura de seleção dos artigos, compondo o desenvolvimento desta revisão integrativa. 
Quadro 1. Quadro expositivo dos artigos pesquisados neste estudo. Rio de Janeiro, RJ, Brasil, 2021.

\begin{tabular}{|c|c|c|}
\hline AUTOR/ANO & TÍTULO & OBJETIVOS \\
\hline Moura, S. F., et al. (2020) & $\begin{array}{l}\text { Padrão Sintomatológico em } \\
\text { Pacientes do Câncer Colorretal de } \\
\text { acordo com a Idade }\end{array}$ & $\begin{array}{l}\text { Investigar a diferença no padrão de sintomas entre } \\
\text { pacientes adultos e idosos com câncer de cólon e } \\
\text { reto. }\end{array}$ \\
\hline Dalmolin, A., et al (2020) & $\begin{array}{l}\text { Saberes e práticas dos profissionais } \\
\text { de enfermagem no cuidado às } \\
\text { pessoas com estoma intestinal }\end{array}$ & $\begin{array}{l}\text { Analisar os saberes e práticas dos profissionais de } \\
\text { enfermagem no cuidado às pessoas com estoma } \\
\text { intestinal de eliminação. }\end{array}$ \\
\hline Bremer, F. P., et al (2020) & $\begin{array}{l}\text { Os imunomarcadores cdx2, beta- } \\
\text { catenina e wnt são úteis para avaliar } \\
\text { a chance de progressão de doença } \\
\text { ou a evolução para óbito em } \\
\text { pacientes com câncer colorretal? }\end{array}$ & $\begin{array}{l}\text { Verificar a existência de relação entre a expressão } \\
\text { de Wnt } 3 \text {, beta-catenina e CDX } 2 \text { em amostras de } \\
\text { câncer colorretal com os eventos clínicos } \\
\text { progressão de doença e óbito. }\end{array}$ \\
\hline Macêdo, L. M., et al (2020) & $\begin{array}{l}\text { Percepções de pacientes } \\
\text { ostomizados com câncer colorretal } \\
\text { acerca da qualidade de vida }\end{array}$ & $\begin{array}{l}\text { O objetivo do estudo foi compreender as } \\
\text { percepções de pacientes afetados por neoplasia } \\
\text { colorretal com estomias acerca da qualidade de } \\
\text { vida. }\end{array}$ \\
\hline Young, A. L. S., et al (2020) & $\begin{array}{l}\text { Avaliação de fatores prognósticos } \\
\text { morfológicos do câncer colorretal } \\
\text { em pacientes do serviço de } \\
\text { oncologia do hospital escola Álvaro } \\
\text { Alvim }\end{array}$ & $\begin{array}{l}\text { O objetivo deste estudo foi realizar a confirmação } \\
\text { dos fatores de prognóstico dos pacientes } \\
\text { diagnosticados com câncer de cólon com a } \\
\text { literatura médica, os quais são: margem de } \\
\text { ressecção circunferencial, invasão linfovascular e } \\
\text { perineural. }\end{array}$ \\
\hline Barboza, M. C. N., et al. (2020) & $\begin{array}{l}\text { Comunicação do diagnóstico de } \\
\text { câncer colorretal à pessoa e família } \\
\text { ou cuidador }\end{array}$ & $\begin{array}{l}\text { Objetivou-se conhecer o impacto da comunicação } \\
\text { do diagnóstico de câncer colorretal à pessoa e sua } \\
\text { família ou cuidador. }\end{array}$ \\
\hline $\begin{array}{l}\text { Correa Júnior, A. J. S., et al. } \\
(2020)\end{array}$ & $\begin{array}{l}\text { Experiências, comportamento } \\
\text { alimentar e características culturais } \\
\text { de pessoas com neoplasias } \\
\text { colorretais }\end{array}$ & $\begin{array}{l}\text { Objetivou-se interpretar } \text { os aspectos } \\
\text { socioculturais, religiosos e espirituais da } \\
\text { experiência de adoecimento de pessoas por câncer } \\
\text { colorretal submetidas ao tratamento cirúrgico por } \\
\text { estomização. }\end{array}$ \\
\hline Salvetti, M. G., et al. (2020) & $\begin{array}{l}\text { Prevalência de sintomas e qualidade } \\
\text { de vida de pacientes com câncer }\end{array}$ & $\begin{array}{l}\text { Analisar a prevalência de sintomas e sua relação } \\
\text { com a qualidade de vida de pacientes com câncer. }\end{array}$ \\
\hline Pacheco-Pérez, L. A., et al. (2019) & $\begin{array}{l}\text { Fatores ambientais } \\
\text { conscientização sobre o câncer } \\
\text { colorretal em pessoas com risco } \\
\text { familiar }\end{array}$ & $\begin{array}{l}\text { Objetivou-se identificar a associação entre os } \\
\text { fatores de risco ambientais e a conscientização } \\
\text { sobre o câncer colorretal em pessoas com risco } \\
\text { familiar. }\end{array}$ \\
\hline Pullig, E. A., et al. (2019) & $\begin{array}{l}\text { Análise do padrão de localização } \\
\text { anatômica do câncer colorretal } \\
\text { no Brasil desde o ano } 2000 .\end{array}$ & $\begin{array}{l}\text { Objetivou-se analisar o padrão de localização } \\
\text { anatômica do câncer colorretal no Brasil, a partir } \\
\text { dos anos } 2000 \text {, considerando ano de notificação, } \\
\text { sexo, faixa etária, topografia e etnia. }\end{array}$ \\
\hline Nascentes, C. C., et al. (2019). & $\begin{array}{l}\text { Rede social no cuidado à pessoa } \\
\text { estomizada por câncer colorretal. }\end{array}$ & $\begin{array}{l}\text { Compreender a influência da rede social no } \\
\text { cuidado de pessoas com estomia por câncer } \\
\text { colorretal e descrever o tipo de apoio que a rede } \\
\text { social oferece para essas pessoas. }\end{array}$ \\
\hline Diger, N. R., et al. (2019) & $\begin{array}{l}\text { Neoplasias intraepiteliais de baixo e } \\
\text { alto graus nas lesões superficiais } \\
\text { colorretais têm maior prevalência } \\
\text { acima de } 65 \text { anos de idade? }\end{array}$ & $\begin{array}{l}\text { Verificar o comportamento das lesões superficiais } \\
\text { do cólon e reto, comparando os achados } \\
\text { anatomopatológicos com os endoscópicos em duas } \\
\text { faixas etárias, abaixo e acima de } 65 \text { anos. }\end{array}$ \\
\hline Reis, P. G. A., et al. (2019) & $\begin{array}{l}\text { Jejum pré-operatório abreviado } \\
\text { favorece realimentação pós- } \\
\text { operatória com menor custo de } \\
\text { internação hospitalar em pacientes } \\
\text { oncológicos }\end{array}$ & $\begin{array}{l}\text { Avaliar a viabilidade de abreviação do jejum em } \\
\text { cirurgias colorretais oncológicas, bem como, o } \\
\text { impacto no desfecho cirúrgico dos pacientes. }\end{array}$ \\
\hline
\end{tabular}




\begin{tabular}{|c|c|c|}
\hline Farias, D. L. S., et al. (2018) & $\begin{array}{l}\text { O enfermeiro como educador em } \\
\text { saúde da pessoa estomizada com } \\
\text { câncer colorretal }\end{array}$ & $\begin{array}{l}\text { Objetivou-se conhecer a experiência de } \\
\text { enfermeiros no processo de educação em saúde } \\
\text { como estratégia de ensino do autocuidado a pessoa } \\
\text { com câncer com estomia intestinal. }\end{array}$ \\
\hline Barba, P. D., et al (2017) & $\begin{array}{l}\text { Demandas de cuidados de pacientes } \\
\text { oncológicos estomizados assistidos } \\
\text { na atenção primária à saúde. }\end{array}$ & $\begin{array}{l}\text { O objetivo desse estudo é evidenciar demandas } \\
\text { de cuidados de pacientes oncológicos } \\
\text { estomizados. }\end{array}$ \\
\hline Valle, T. D., et al. (2017) & $\begin{array}{l}\text { Fatores intervenientes para o início } \\
\text { do tratamento de pacientes com } \\
\text { câncer de estômago e colorretal }\end{array}$ & $\begin{array}{l}\text { Objetivou-se identificar o tempo entre os } \\
\text { sintomas, a busca por assistência e o início do } \\
\text { tratamento em pacientes com câncer de estômago } \\
\text { e colorretal e os fatores que interferem nesses } \\
\text { processos. }\end{array}$ \\
\hline Ribeiro, S. M., et al. (2017) & $\begin{array}{l}\text { Efeitos da suplementação de zinco } \\
\text { na fadiga e na qualidade de vida de } \\
\text { pacientes com câncer colorretal }\end{array}$ & $\begin{array}{l}\text { Objetivou-se investigar os efeitos da } \\
\text { suplementação oral de zinco sobre a intensidade da } \\
\text { fadiga e a qualidade de vida de pacientes durante a } \\
\text { quimioterapia para neoplasia colorretal. }\end{array}$ \\
\hline Mesquita, M. E. R., et al/ 2017 & $\begin{array}{l}\text { Autocuidado e Quimioterapia Oral } \\
\text { Domiciliar: Avaliação das Práticas } \\
\text { Educativas dos Enfermeiros sob a } \\
\text { Perspectiva de Pacientes }\end{array}$ & $\begin{array}{l}\text { Avaliar, na perspectiva dos pacientes, } \\
\text { convergências e divergências entre orientações } \\
\text { para o autocuidado, compreensão quanto às } \\
\text { orientações e sua aplicação. }\end{array}$ \\
\hline Hardiman, K. M., et al. (2016) & $\begin{array}{l}\text { A lista de verificação de autocuidado } \\
\text { centrada na autonomia do paciente } \\
\text { reduz as readmissões hospitalares } \\
\text { após a criação da ileostomia. }\end{array}$ & $\begin{array}{l}\text { Projetar e implementar uma lista de verificação } \\
\text { centrada no paciente, promovendo independência } \\
\text { e validando o conhecimento e as habilidades de } \\
\text { autocuidado e avaliamos seu efeito nas } \\
\text { readmissões após a criação da ileostomia. }\end{array}$ \\
\hline Santos, S. C. D., et al. (2016) & $\begin{array}{l}\text { Doença de Crohn: fator de risco } \\
\text { para o carcinoma colorretal }\end{array}$ & $\begin{array}{l}\text { Objetivou-se descrever os mecanismos } \\
\text { responsáveis pelo aumento do risco de carcinoma } \\
\text { colorretal subjacentes à doença de Crohn. }\end{array}$ \\
\hline
\end{tabular}

Fonte: Dados da pesquisa.

Os resultados obtidos através dos 20 artigos científicos selecionados, trouxeram efetivação a elaboração de três categorias temáticas: 1- Os principais cuidados de enfermagem ao paciente com câncer colorretal; 2- Qualidade de vida dos pacientes ostomizados portadores de câncer colorretal; e 3- A importância da educação em saúde aos pacientes com câncer colorretal.

\section{Categoria 1- Os principais cuidados de enfermagem ao paciente com câncer colorretal}

O diagnóstico de uma doença grave, com alta morbidade e mortalidade, como o câncer colorretal, acarreta sofrimento físico e emocional à pessoa doente e seus familiares e/ou cuidadores. Esse processo é vivenciado como um momento de angústia, rodeado por estigmas sociais que estão associados a um tratamento mutilador, doloroso e letal. Nessa perspectiva, comunicar más notícias, como o diagnóstico de câncer, torna-se um evento delicado, e por isso, a forma como é passada a informação é fundamental no intuito de minimizar o sofrimento atribuído a essa malignidade. Neste intuito, a qualidade da comunicação por parte do profissional deve estar embasada em um atendimento de qualidade, conferindo apoio emocional, orientações, auxílio e confiança para lidar com as adversidades impostas pelo adoecimento. A comunicação de más notícias necessita ser aplicada mediante capacitação e treinamento entre todos os profissionais envolvidos no contexto do adoecimento, uma vez que a forma utilizada para transmitir este diagnóstico é de extrema importância para promover melhor aceitação e enfrentamento de uma doença grave, como o câncer. (Barboza, et al. 2020)

Os cuidados de enfermagem não se restringem em apenas executar técnicas ou procedimentos, mas também em ações de cuidados abrangentes, trazendo outros aspectos e dentre eles desenvolver a habilidade de comunicação. Deste modo, o uso da comunicação como instrumento básico do enfermeiro é um meio utilizado para atender as necessidades do paciente. 
De acordo com o estudo de Farias, et al (2018), o tratamento do câncer colorretal está baseado em processos quimioterápico e radioterápico, porém se destaca à cirurgia como sendo o principal tratamento, acredita-se em uma possibilidade efetiva do tratamento a ressecção da porção do intestino afetado, na qual se torna indicado a confecção de uma estomia, que na maioria dos casos assume caráter definitivo e uma outra precaução é que não ocorra a metástase para outros órgãos. A prevenção deste câncer está ancorada em dois aspectos principais, ou seja, a prevenção primária vinculada à educação e modificação do estilo de vida, e, a secundária, que consiste na detecção precoce, por meio da identificação de sinais e sintomas. Neste sentido o enfermeiro no processo do cuidar integra ao processo assistencial a pessoa com doença crônica, como o câncer colorretal, e com estomia o espaço em ser o centro desse processo, desempenhando um papel ativo e particípio da sua reabilitação, sendo assim, ressaltamos o papel do enfermeiro no processo do cuidar, como sendo o profissional que participa de todo o processo de reabilitação, o qual deve ocorrer com dinamismo para que seja enfrentado as problemáticas da aceitação, adaptação e principalmente na realização do autocuidado no domicílio. (Valle, et al. 2017)

Os pacientes oncológicos carecem de afeto e o contato assíduo com a equipe de enfermagem compartilhando de sentimentos e emoções, podendo resultar em empatia, o envolvimento favorece a empatia e essa se torna essencial quando se propõe a ajudar alguém, o papel do enfermeiro na reabilitação oncológica se volta para o ensino, aconselhamento e orientação o que faz com que o mesmo se torne essencial na ação do cuidar, dele é exigida a capacidade de desenvolver a educação ao paciente e ao familiar, em alguns casos, após a remoção cirúrgica do tumor, é necessário exteriorizar o cólon realizando a estomia, o paciente, dependendo do estadiamento da doença e do tratamento realizado, pode utilizar provisória ou permanentemente uma bolsa especial que auxilia no processo de evacuação das fezes onde cabe ao enfermeiro a orientar quanto a alimentação adequada, como também a manter a higiene adequada da bolsa de colostomia.

Dor e fadiga são os sintomas mais prevalentes em pacientes com câncer, segundo Salvetti, et al. (2020), uma das principais queixas dos pacientes que são submetidos a tratamentos quimioterápicos é a fadiga, que em doenças avançadas, pode ser descrita como cansaço, fraqueza ou falta de energia. A fadiga é um sintoma angustiante que ocorre em 39 a $90 \%$ dos pacientes sob quimioterapia, de forma a afetar o funcionamento físico, emocional e/ou cognitivo. A intensidade da fadiga aumenta com os ciclos consecutivos de quimioterapia, pode reduzir a adesão ao regime terapêutico planejado e tem um impacto negativo nas diferentes dimensões de qualidade de vida. A deficiência de zinco está associada à intensidade da fadiga na síndrome da fadiga crônica, relacionada aos marcadores de inflamação e ativação imunológica, além de ao estresse oxidativo associado ao dano à membrana lipídica. Estudos já apontam que a suplementação do zinco preveniu a piora da fadiga e da qualidade de vida em pacientes submetidos à quimioterapia após cirurgia para câncer colorretal. (Ribeiro, et al. 2017)

É possível compreender que, com estudo e interesse, o profissional de enfermagem é capaz de dar todas as orientações necessárias ao paciente, inclusive as nutricionais, visto que fará parte do processo de autocuidado do mesmo a partir da alta hospitalar. Diante da necessidade de compartilhar as suas experiencias, o enfermeiro pode trazer aos pacientes como forma de cuidado, pesquisas e matérias sobre o assunto ao qual o paciente vivencia como também indicar redes físicas e sociais de apoio a esses pacientes, o contato com outras pessoas que vivenciam com o mesmo grau de dificuldade traz ao paciente uma expectativa de melhoria e adaptação de vida esse é um tipo de cuidado que traz muito benefício psicológico pois através deste o indivíduo consegue se inserir à sociedade de forma que não se sinta diferente.

Ressalta-se que a prevenção do câncer colorretal é uma área de desenvolvimento pouco explorada pelo profissional de enfermagem que trabalha em diferentes contextos, devido às poucas evidências encontradas na América Latina sobre estudos liderados por enfermeiros e que incluam o componente genético e hereditário do câncer colorretal. (Pacheco-Pérez, et al. 2019)

De fato, concordamos que a prevenção é pouco explorada pelo profissional de enfermagem, porém cabe ressaltar que a prevenção é um cuidado primordial a todos os indivíduos e que a enfermagem tem um papel muito importante em trazer a realidade da população informações sobre a doença como também sobre exames para rastreio, orientar aos indivíduos durante a 
consulta de enfermagem sobre esse tipo de câncer explicando de forma que as pessoas entendam a gravidade e busquem a prevenção.

\section{Categoria 2- Qualidade de vida dos pacientes ostomizados portadores de câncer colorretal.}

Segundo Valle, et al. (2017), a depender do estadiamento da neoplasia colorretal, o principal tratamento é o cirúrgico, de forma que a cirurgia mais radical implica a remoção do intestino grosso e reto, com necessidade de confecção de estomia, com impacto social, físico e psicológico, tendo como meta contornar complicações procedentes de neoplasias e doenças intestinais. A palavra estomia tem origem grega, "stomia" que significa "abertura", a qual é procedida, principalmente, no trato gastrointestinal, cujos principais tipos são as que possuem função de eliminação intestinal, sendo denominadas colostomias e ileostomias. Apesar dos benefícios da estomia, esse procedimento acarreta muitas alterações na vida da pessoa, podendo ser no âmbito social, psicológico ou religioso. O indivíduo necessita de harmonização das tarefas do dia a dia, cuidados pós-operatórios, autocuidado e interações sociais. Além disso, há o desafio da aceitação da autoimagem, o sentimento de luto e as mudanças nos hábitos de vida, devido ao estoma. Ao considerar os impactos na vida dos indivíduos que vivem com estomias, provenientes do câncer colorretal, é fundamental conhecer a subjetividade que permeia tais alterações e como estas impactam na qualidade de vida dessas pessoas. Nessa situação, o uso da avaliação da qualidade de vida deve permitir compreender mudanças, permeando do estado psicológico até o meio ambiente em que pessoas estomizadas vivem. (Macêdo, et al. 2020)

De acordo com o estudo de Correa Júnior, et al. (2021), o processo de adoecimento e posterior estomização gera repercussões fisiológicas como liberação de gases e de ruídos desagradáveis, além da fadiga, culminando em repercussões psicossociais como depressão e insatisfação com a aparência pela utilização de equipamentos coletores. Nesse sentido, a ação da enfermagem é de inteirar-se das necessidades psicoemocionais orientando os familiares acerca do tratamento e vida após a estomização. A humanização do cuidado é produto das atividades técnicas, do conhecimento científico e do expressivo envolvimento do profissional nas ações do cuidar e das relações interpessoais presentes nesse processo. Para o cuidado da pessoa que vivencia a confecção cirúrgica de um estoma, é imprescindível que este seja pautado em uma visão holística que contemple o humanismo nas ações do cuidar e considere as especificidades necessárias para a adaptação e a reabilitação dessa pessoa. (Dalmolin, et al. 2020)

Ao entender que a ostomia consiste no ato cirúrgico que exterioriza parte do órgão para a parede abdominal, deixando assim, o mesmo, exposto, entende-se também que independente do tempo de uso da ostomia, podendo ser provisória ou definitiva, é importante o enfermeiro ter em mente que este indivíduo terá que se adaptar a um novo estilo de vida, o que pode acarretar mudanças bruscas de comportamento e humor, visto que o mesmo poderá vir a sentir desconforto, vergonha, receio e principalmente incômodo com o fato de não ter controle sobre uma área específica de seu corpo. Como dito, tal fato reflete em todas as áreas da vida deste paciente, e uma área extremamente afetada será a psicológica, fazendo com que este paciente se sinta constrangido, o que afetará diretamente suas relações interpessoais e dificultará no processo do cuidado, portanto a atenção da enfermagem precisa ser redobrada.

Acredita-se que a rede e o apoio social assumem papel relevante no enfrentamento das dificuldades decorrentes das doenças crônicas e no sucesso do tratamento. Entende-se o termo rede social como um conjunto de relações interpessoais que determinam as características da pessoa, tais como: hábitos, costumes, crenças, valores; sendo que desta rede a pessoa pode receber apoio do tipo emocional, material e informativo ou pode receber influências negativas, uma vez que nem sempre a presença de vários membros da rede social na vida de uma pessoa resulta em efeitos emocionais e ou comportamentais positivos. Qualifica-se rede social em duas grandes categorias: rede primária e rede secundária, sendo a rede primária, relação entre os familiares, amigos, vizinhos ou colegas, e a rede secundária pela relação que a pessoa estabelece com membros das instituições públicas de assistência à saúde, educação, assistência social ou outras. Neste estudo, compreende-se que os membros da rede 
social primária e secundária exercem forte influência no cuidado às pessoas com estomia devido ao câncer colorretal, sendo os membros da família, especialmente dos filhos, irmãos e cônjuges, como essenciais para o acompanhamento dos participantes durante a busca pelos serviços de saúde e no período em que estes se encontravam em seus domicílios. Os mesmos ofereceram apoio emocional, material e nos cuidados cotidianos, o que influenciou na autoestima e no enfrentamento das situações adversas. Constatou-se também que o apoio dos amigos caracterizado pela presença, afeto, preocupação, auxílio financeiro, palavra de conforto e de fé, contribuíram para o alívio e bem-estar espiritual das pessoas com estomia, gerando força e tranquilidade na vivência cotidiana, além da relação com os profissionais dos serviços dos níveis secundários e terciários de atenção à saúde que foi marcada pelo acolhimento, orientação e suporte material, enquanto a relação com os profissionais de unidades básicas de saúde se mostrou fragilizada, devido à falta de vínculo, preparo e disponibilidade desses profissionais para o acompanhamento do tratamento do câncer e cuidados com a estomia.

Concluiu-se que o conhecimento da rede social da pessoa com câncer colorretal constitui em um importante subsídio para que profissionais de todos os níveis de atenção, possam promover uma assistência integral, articulada, resolutiva e um autocuidado apoiado. (Nascentes, et al. 2019)

É possível entender que ainda existem obstáculos com o manejo de pacientes oncológicos por parte dos profissionais de saúde da Atenção Primária. É preciso melhorar os níveis de conhecimento e capacitação dos profissionais que futuramente terão que lidar com estes pacientes e principalmente os que já atuam com os mesmos, para que se sintam acolhidos e seguros com o processo, principalmente em relação ao autocuidado, o qual o enfermeiro, precisa acompanhar. Estes profissionais precisam se conscientizar do cuidado ao paciente como um todo e não somente à doença, uma vez que este paciente pode não desfrutar de uma rede social ideal por viver sozinho, não ter apoio familiar ou nenhum outro membro com quem possa contar.

Nessa situação, o profissional precisa ser mais cauteloso e acolhedor com este paciente, pois uma vez que existe este vínculo, existe também a confiança, e o paciente se sente mais confortável, seguro e responsável na hora de ir para casa e coloca em prática o autocuidado. É importante também que o profissional se mantenha atualizado, principalmente para saber que hoje em dia, por exemplo, existem pessoas ostomizadas em redes sociais na internet que mostram que um paciente com este acometimento pode levar uma vida normal, além de quebrar diversos preconceitos e desconhecimento em relação à doença e à ostomia. Isso, para alguns pacientes pode ser um alívio, por saber que existem outras pessoas nas mesmas condições, se expondo para tirar dúvidas e quebrar paradigmas da sociedade, demonstrando total apoio aos semelhantes.

Segundo Barba, et al. (2017), a pessoa com estomia estabelece, com a Atenção Primária ou Atenção Básica, o primeiro contato, fora do ambiente hospitalar, com o serviço de saúde. Cabe aos profissionais desta acolhê-los de forma humanizada e resolutiva, com vistas à integralidade da atenção. Estas equipes de saúde, preferencialmente multidisciplinares, necessitam estar preparadas, por meio do planejamento da assistência e da educação em saúde, para desenvolver as aptidões do indivíduo para o autocuidado, bem como apoiá-lo em diferentes demandas de cuidado. O enfermeiro da Atenção Básica é o que mais se aproxima e está em contato com o paciente em seu domicílio, conhecendo seus hábitos de vida, condições socioeconômicas e culturais, aspectos estes que favorecem o cuidado. Devido à significativa incidência de estomias relacionadas ao CA de cólon e reto, tornase fundamental ampliar os conhecimentos acerca desta realidade entre os profissionais da saúde, sociedade e os próprios estomizados, para que adquiram maior esclarecimento e segurança, com vistas a melhorar a qualidade de vida.

Sabe-se que a enfermagem é responsável pela educação em saúde e atuante na prevenção primária ao câncer, uma vez que o mesmo, na consulta de enfermagem, possui respaldo para educar e estimular o paciente a adotar um estilo de vida saudável, principalmente quando se trata de hábitos alimentares e orientação nutricional juntamente com outros profissionais da equipe. Já existem estudos que falam sobre a alimentação do paciente portador de câncer no trato gastrointestinal, sobre a ingestão de carnes vermelhas e processadas, como fator de risco para este tipo de patologia, além do sedentarismo, dos vícios e maus hábitos que facilitam o desenvolvimento dessa e outras doenças. Com o atendimento sempre individualizado, buscando adaptação e aceitação do paciente ostomizado, o enfermeiro precisa estimular o autoconhecimento neste e garantir que o mesmo não se isole 
e siga inserido no contexto social, vivendo sua vida sem que a mesma gire em torno da doença e da bolsa coletora, mostrando que ele pode ter qualidade em todos os âmbitos de sua vida, seja profissional, sexual, psicológica, social e pessoal. Cabe à enfermagem buscar maiores subsídios para respaldar suas intervenções e diminuir as estatísticas dessas doenças, de acordo com a procura da população para promoção da saúde e prevenção de doenças.

\section{Categoria 3- A importância da educação em saúde aos pacientes com câncer colorretal.}

A educação em saúde é uma das atribuições imprescindíveis do enfermeiro no processo de cuidar da saúde aos pacientes com câncer colorretal. O tratamento do câncer colorretal está baseado em processos quimioterápicos e radioterápicos, porém se destaca a cirurgia como sendo o principal tratamento. As pessoas com estomia intestinal necessitam de fortalecimento no seu autocuidado para sua recuperação e reabilitação. Assim, o enfermeiro possui o papel fundamental no ensino do paciente com estomia intestinal e sua família para o desenvolvimento da autonomia. É imprescindível que o enfermeiro acolha e oriente a pessoa e sua família em conexão com a educação em saúde de forma eficiente e eficaz para a manutenção da qualidade de vida e desta forma privilegiando o desenvolvimento de competências para o autocuidado.

De acordo com Farias, et al. (2018), quando se promove o acesso à informação, observa-se a adaptação das dificuldades na aceitação da autoimagem e o ganho da autonomia na realização do autocuidado.

É necessário que o profissional de enfermagem busque junto a família e todas as informações estruturais que dispõe o paciente acometido por essa patologia, não apenas resumir o tratamento técnico somente dentro da unidade hospitalar, mas sim buscar educar tanto o paciente quanto a todos que diretamente irão estar com ele, pois o contrário, seria entregar o paciente a própria sorte. Para que isso não ocorra, a equipe de enfermagem deve estar atenta a todas as informações que o paciente possa disponibilizar, como por exemplo quantidade de integrantes da família e quem diretamente o acompanha, é de bom tom que mais pessoas ligadas ao paciente diagnosticado com o câncer colorretal estejam envolvidos em seu tratamento e saibam que essa é uma doença hereditária.

A presença do estoma, associada à bolsa coletora, as mudanças nos padrões de eliminação, dos hábitos alimentares e de higiene vêm a calhar em sentimentos como medo, incertezas e preocupações, eventos estes que podem comprometer a reabilitação destes pacientes. Com a alta hospitalar, o estomizado, no domicílio, depara-se com a nova realidade de vida. Nessa perspectiva, os profissionais na área da saúde necessitam desenvolver ações de educação em saúde, no sentido de contribuir para que o estomizado adquira autonomia para seu autocuidado. Nesse sentido, os profissionais da Atenção Básica necessitam estimular o autocuidado, potencializando a assistência em suas necessidades biopsicossociais, entre elas, a manutenção da bolsa de ostomia. Após a realização da estomia, uma das preocupações dos estomizados é com as eliminações intestinais. Desse modo, o paciente necessita ser orientado, ensinado e treinado para assumir o seu autocuidado. Para tanto, os mesmos buscam alternativas para evitar ao máximo a possibilidade de vazamento do seu conteúdo. O dispositivo deve ser esvaziado sempre que o conteúdo atingir um terço ou, no máximo, a metade de sua capacidade, para aumentar sua durabilidade. Cabe ainda, ao paciente, observar se a bolsa ficou bem fechada, para evitar acidentes. Não deverá ser aplicado nenhum produto na pele, a menos que tenha sido indicado por um profissional. Além disso, se recomenda que a troca da bolsa coletora seja feita durante o banho, com água morna, evitando jato forte e com o uso de sabão neutro. A secagem deve ser realizada com um tecido macio com uso específico para este fim. Na troca da bolsa, é importante que o paciente realize a inspeção de seu estoma, bem como da pele em seu entorno. O orifício do estoma saudável é caracterizado pela cor rósea ou vermelho vivo, úmido, de formato nem sempre regular. A pele ao redor deve estar lisa, sem hiperemia, prurido, lesões ou dor. Sua protusão deverá medir aproximadamente $1,5 \mathrm{~cm}$, externamente ao abdome. A utilização de substâncias como álcool, benzina, colônias, tintura de benjoim, mercúrio, mertiolate, pomadas e cremes no periestoma poderá comprometer sua integridade, causando ressecamento, lesões e mesmo reações alérgicas, além de impedir a adaptação do coletor, o que pode ocasionar vazamento. A realização da consulta de Enfermagem, atividade privativa deste profissional, constitui-se uma ferramenta importante no retorno do estomizado ao domicílio, pois, por meio desta, possibilita-se conhecê-lo, avalia-lo clinicamente e indicar os dispositivos adequados, de acordo com suas 
necessidades biopsissociais. Para a escolha adequada da bolsa, o enfermeiro deve considerar sua localização, tipo e tamanho do estoma, característica e quantidade de drenagem, tamanho e contorno do abdome, condição da pele (periestoma), atividades físicas, destreza e idade do indivíduo. Na prática da Enfermagem, educação e cuidado devem interligar-se e o enfermeiro, para além de ser um cuidador, é um educador em saúde.

De acordo com Barba, et al. (2017), nessa medida, é essencial que esse profissional esteja preparado e utilize a educação em saúde como instrumento para a assistência efetiva, promovendo ações que incentivem o autocuidado e possibilitem, aos pacientes e/ou familiares, tornarem-se multiplicadores dos conhecimentos adquiridos. Dentre estas ações, que incluem assistência integral relativa ao cuidado, destacam-se as estratégias de instrumentalização por meio de palestras, seminários, distribuição de materiais educativos, grupos de apoio e avaliações de saúde. A aplicabilidade dessas ferramentas tem potencial de incentivá-lo a tornar-se protagonista de seu autocuidado no domicílio, contando com apoio profissionais.

De fato, um grande desafio a ser enfrentado pelo profissional de enfermagem, quanto ao seu papel de educar, é justamente a falta de estudos com pautas voltadas ao treinamento em educação do portador de câncer colorretal e seus familiares. Será preciso ter disciplina e interesse em desenvolver cada vez mais esse tema, de acordo com a complexidade e a grande demanda quando se fala em cuidados de enfermagem e educação em saúde que o profissional precisa dominar para educar o paciente e seus familiares na continuidade do que foi ensinado. E para melhor educar o paciente e todos diretamente envolvidos com o mesmo, é necessário entender bem, e de forma individualizada suas condições socioeconômicas. Isso dará um caminho ao profissional de enfermagem na melhor abordagem, personalizada e de acordo com as condições reais do paciente que dele dependem os cuidados a partir da alta, fora das condições da unidade hospitalar.

O estudo de Valle, et al. (2017), mostra que o câncer colorretal enquadra-se como uma neoplasia de alta incidência e prevalência no mundo, sendo esta a quarta, e no Brasil, a quinta, demonstrando assim a atualidade da pauta e a importância de elaborar estudos a respeito do tema em questão. Dessa forma, reforça-se a necessidade da realização de investigações similares nas demais regiões do país, produzindo estudos que permitirão identificar barreiras e soluções, contribuindo para a elaboração de uma política pública brasileira bem estruturada, para o atendimento dos potenciais casos de câncer de estômago e colorretal. A utilização de linguagem técnica ou científica foi identificada pelos pacientes e seus familiares e/ou cuidadores como um fator prejudicial à comunicação com os profissionais de saúde. (Barboza, et al. 2020)

É nítida a falta de busca e especialização na área da estomaterapia e na atenção especial em todos os aspectos que o profissional precisa ter com esse paciente. Precisamos urgentemente de profissionais especializados e mestres que possam apresentar aos futuros profissionais, quais os cuidados e as orientações que esse paciente ostomizado precisa ter ao sair da unidade hospitalar e chegar ao seu lar.

\section{Conclusão}

Após a pesquisa, leitura e desenvolvimento das categorias, conseguimos destacar a importância do cuidado de enfermagem e do enfermeiro como educador em saúde. Sabemos que a medicina não possui o toque e o cuidado, característicos da profissão de enfermagem, então é crucial que ela seja mantida, principalmente quando se trata de um paciente ostomizado que sofreu uma mudança brusca no estilo de vida. O enfermeiro que lidar com este paciente, precisará alcançar o maior nível de empatia e ter uma sensibilidade na escuta que ninguém será capaz de suprir, pois como vimos, será de extrema importância existir um vínculo entre profissional e paciente, assim como o de professor e aluno. Assim que o enfermeiro entende o seu papel de educador e passa segurança ao paciente, o processo de confiança é rápido e natural, fazendo assim com que o mesmo sempre o procure quando precisar de uma assistência.

Mais uma vez, conseguimos observar a importância da Sistematização da Assistência de Enfermagem, que se seguida com atenção e responsabilidade, é possível prestar um atendimento de qualidade, principalmente visando o paciente como um 
todo, e não só a doença que ele porta, não se atentando só as técnicas, mas também as orientações e acompanhamento do mesmo. Se atentar à todas as etapas da sistematização e preparar este paciente desde a descoberta da doença, o pré-operatório, até a alta hospitalar e ida para casa, sempre estimulando o autocuidado.

Vimos também como as redes de apoio são importantes e como os profissionais podem ser cruciais até neste quesito, pois existem pessoas que podem estar nessa situação e não ter rede de apoio nenhuma, apenas o vínculo com o profissional. Pensando nisso, é preciso criarmos redes de apoio sociais voltadas aos pacientes ostomizados, encontros, palestras, ações pedagógicas tanto para os portadores, familiares dos portadores, quanto para profissionais que atuam na área e os que não atuam, mas tem interesse também. É preciso de uma rede de profissionais especializados e capacitados para prestar um atendimento satisfatório a esses pacientes, que possam suprir suas necessidades em todos os âmbitos: psicológico, físico, nutricional, social, etc. E por fim, mostrar que pacientes ostomizados não estão sozinhos, que os mesmos podem viver suas vidas sem medo ou vergonha, com independência e com controle de sua doença.

Pela dimensão do país e muitas diferenças culturais de uma região em comparação a outra, como sugestão para pesquisas futuras, torna-se necessário a adaptação da prática de educação em saúde oferecida pela equipe de enfermagem envolvida no cuidado do paciente diagnosticado e em tratamento do câncer colorretal. Fatores que implicam para uma educação mais eficaz é a linguagem que o profissional de enfermagem irá utilizar, pois é certo que poucas pessoas dominam o entendimento de termos técnicos utilizados na educação em saúde, e isso pode afastar o paciente do melhor entendimento e assim não atingir o objetivo esperado, causando frustração e atrapalhando o processo do cuidado.

\section{Referências}

Barba, P. D., et al. (2017). Demandas de cuidados de pacientes oncológicos estomizados assistidos na atenção primária à saúde. Rev. enferm. UFPE on line 11(8): 3122-3129, https://pesquisa.bvsalud.org/portal/resource/pt/bde-32536

Barbosa, E., (2021). Proposições sobre a ressignificação do cuidado de Enfermagem: um estudo teórico-reflexivo. Enfermagem em Foco. http://revista.cofen.gov.br/index.php/enfermagem/article/view/4188/1017>. https://doi.org/10.21675/2357-707X.2020.v11.n5.4188.

Barboza, M. C.N., et al. (2020). Comunicação do diagnóstico de câncer colorretal à pessoa e família e/ou cuidador. Journal Health NPEPS. 5(2): 226-239. https://periodicos.unemat.br/index.php/jhnpeps/article/view/4585

Brasil. (2021). Ministério da Saúde. Tipos de câncer: Câncer de intestino - versão para Profissionais de Saúde. https://www.inca.gov.br/tipos-de-cancer/cancerde-intestino/profissional-de-saude

Bremer, F. P., et al. (2020). Os imunomarcadores cdx2, beta-catenina e wnt são úteis para avaliar a chance de progressão de doença ou a evolução para óbito em pacientes com câncer colorretal? ABCD Arq Bras Cir Dig 2020;33(3):e1534 https://doi.org/10.1590/0102-672020200003e153

Correa Júnior, A.J. S., et al. (2021). Experiências, comportamento alimentar e características culturais de pessoas com neoplasias colorretais. Rev Bras Enferm;74(1):e20200092 http://dx.doi.org/10.1590/0034-7167-2020-0092

Dalmolin, A., et al. (2020). Saberes e práticas dos profissionais de enfermagem no cuidado as pessoas com estoma intestinal. Rev. Bras. Enferm. 73 (suppl5) • https://doi.org/10.1590/0034-7167-2020-0018

Diger, N. R., et al. (2019). Neoplasias intraepiteliais de baixo e alto graus nas lesões superficiais colorretais têm maior prevalência acima de 65 anos de idade. $A B C D$, arq. bras. cir. dig. $32(04) \cdot$ https://doi.org/10.1590/0102-672020190001e1478

Farias, D. L. S., et al. (2018). O enfermeiro como educador em saúde da pessoa estomizada com câncer colorretal. Enferm. Foco 10 (1): 35-39 http://revista.cofen.gov.br/index.php/enfermagem/article/view/1486/490

Macêdo, L. M., et al. (2020). Percepções de pacientes estomizados com câncer colorretal acerca da qualidade de vida. Rev Rene. DOI: 10.15253/21756783.20202143946 http://repositorio.ufc.br/handle/riufc/53168

Mesquita, M. E. R., et al. (2016). Autocuidado e quimioterapia oral domiciliar: Avaliação das práticas educativas dos enfermeiros sob a perspectiva dos pacientes. Revista Brasileira de Cancerologia; 62(3): 237-245. http://www1.inca.gov.br/rbc/n_62/v03/pdf/06-artigo-autocuidado-e-quimioterapia-oral-domiciliaravaliacao-das-praticas-educativas-dos-enfermeiros-sob-a-perspectiva-de-pacientes.pdf

Moura, S. F., et al. (2020). Padrão Sintomatológico em Pacientes do Câncer Colorretal de acordo com a Idade. Revista Brasileira de Cancerologia; 66(1): e15474. https://doi.org/10.32635/2176-9745.RBC.v66n1.474

Nascentes C.C., et al. (2019). Rede social no cuidado à pessoa estomizada por câncer colorretal. Rev. enferm. UFPE on line ; 13: [1-7]. https://pesquisa.bvsalud.org/portal/resource/pt/biblio-1046359

Pacheco-Pérez, L. A., et al. (2019). Fatores ambientais e conscientização sobre o câncer colorretal em pessoas com risco familiar. Rev Lat Am Enfermagem 27 • https://doi.org/10.1590/1518-8345.3082.3195 
Research, Society and Development, v. 10, n. 9, e46910918281, 2021

(CC BY 4.0) | ISSN 2525-3409 | DOI: http://dx.doi.org/10.33448/rsd-v10i9.18281

Pullig, E. A., et al. (2019). Análise do padrão de localização anatômica do câncer colorretal no Brasil desde o ano 2000. Centro Universitário de Anápolis Unievangélica Curso de Medicina - Anápolis - Goiás. https:repositorio.aee.edu.br > bitstream > aee

Reis, P. G. A., et al. (2019). Jejum pré-operatório abreviado favorece realimentação pós-operatória com menor custo de internação hospitalar em pacientes oncológicos. Rev. Col. Bras. Cir. 46 (3) •https://doi.org/10.1590/0100-6991e-20192175

Remor. A., et al. (1986). A teoria do auto cuidado e sua aplicabilidade no sistema de alojamento conjunto. Rev. Bras. Enf [BrasJ1ia, 39(2/3):

Ribeiro, S. M., et al. (2017). Efeitos da suplementação de zinco na fadiga e na qualidade de vida de pacientes com câncer colorretal. Einstein (São Paulo) 15 (1) • https://doi.org/10.1590/S1679-45082017AO3830

Salvetti, M. G., et al. (2020). Prevalência de sintomas e qualidade de vida de pacientes com câncer. Rev. Bras. Enferm. 73 (2) • https://doi.org/10.1590/00347167-2018-0287

Santos, S. C. D., (2017). Doença de Crohn: fator de risco para o carcinoma colorretal. J. Coloproctol. (Rio J.) 37 (1) • https://doi.org/10.1016/j.jcol.2016.06.005

Valle, T. D., et al. (2017). Fatores intervenientes para o início do tratamento de pacientes com câncer de estômago e colorretal. Rev. Latino-Am. Enfermagem Artigo Original 2017;25:e2879 10.1590/1518-8345.1493.2879 https://www.scielo.br/j/rlae/a/VqbXv3GwM4WJS6qtj9wVKKd/?lang=pt\&format=pdf

Young, A. L. S., et al. (2020). Avaliação de fatores prognósticos morfológicos do câncer colorretal em pacientes do serviço de oncologia do hospital escola Álvaro Alvim. Revista Cientifica da FMC. 15, http://fmc.br > ojs > RCFMC > article > download 